\title{
Overview of Allopurinol Decisions in Primary Care: A Narrative Review ${ }^{1}$ Multag Jaual Alqahtani, ${ }^{2}$ Abdullah Mohammad Alshamrani \\ ${ }^{1}$ Primary Care Unit, Jubail General Hospital, ${ }^{2}$ Health Care Center, Ministry of Health, Khobar, KSA Corresponding author: Mutlaq Alqahtani ,E-mail: mutlaq.j@gmail.com, Tel no: +966536565683
}

\begin{abstract}
Background: Allopurinol is a type of drug called a xanthine oxidase inhibitor. It functions by lowering the production of uric acid in the body. It can be found in the kind of an oral tablet. Generalist physicians, specifically general internists and primary care physicians, are usually the first to see individuals with gout and for that reason play an essential role in the diagnosis and management of these patients. Gout is mostly managed in primary care and lasting treatment aims to reduce serum uric acid degrees with using allopurinol. Urate lowering therapy is indicated in patients who have had an acute gout attack and whose uric acid level remains high, increasing the risk of a subsequent attack. The effect of allopurinol on uric acid level is dose dependent and variable. Aim of the Study: In the present review, we aimed at discussing the indications of Allopurinol in primary care along with its benefits and side effects which should be considered by patients and physicians before prescribing it. Methods: Narrative review was conducted using electronic database such as; PubMed/Medline, Embase, Google scholar, for relevant articles related to our concerned topic which is about the usage of allopurinol in primary care and methods that family doctors use to make a decision about the usage, published up to 2018. Conclusion: The benefit-risk balance of allopurinol should be carefully assessed with respect to the patients' condition and medical profile before prescription in the PCU.
\end{abstract}

Keywords: Allopurinol, Primary Healthcare, xanthine oxidase inhibitors.

\section{INTRODUCTION}

Allopurinol, a xanthine oxidase inhibitor, is one of the most frequently utilized hypouricemic agents. It is generally utilized for lasting control of gout, prevention of tumor lysis syndrome, and the control of hyperuricemia in chronic kidney illness. Moreover, its valuable duty as an antioxidant suggests a potentially novel use for this drug ${ }^{[1]}$. In spite of its reliable hypouricemic result, allopurinol can create adverse responses in $2 \%$ to $8 \%$ of users, ranging from cutaneous hypersensitivity responses to the possibly serious allopurinol hypersensitivity syndrome (AHS), which causes fatality in up to $27 \%$ of cases ${ }^{[2]}$. Allopurinol is reported to be a typical source of Stevens-Johnson syndrome and complex renal failure ${ }^{[3]}$.Allopurinolinduced unfavorable reactions have been reported to develop in organization with inappropriately high dosage, renal insufficiency, concurrent use diuretics, and the existence of the HLA-B * 5801 allele ${ }^{[4]}$. Accumulation of oxipurinol, a major metabolite of allopurinol, was suggested as a feasible reason for the negative responses, and a guideline of dosage adjustment according to creatinine clearance has been suggested ${ }^{[5]}$. However, many researches on the risk elements for allopurinol generated unfavorable occasions have concentrated on a minimal set of threat variables, and several recent research studies could not validate these previous threat elements.

In this review we discuss the usage of allopurinol in primary care, it benefits and side effects which should be concerned by patient and doctor before prescribing it.

\section{METHODS}

The present narrative review was conducted using electronic database such as; PubMed/Medline, Embase, Google scholar, for relevant articles related to our concerned topic which is about the usage of allopurinol in primary care and methods that family doctors use to make a decision about the usage, published up to 2018. Restriction were applying to only English language articles with human subject.

\section{Allopurinol Decisions in Primary Care \\ - The Relationship between Uric acid, Gout and Allopurinol}

Generalist physicians, especially general internists and primary care physicians, are frequently the very first to see patients with gout and consequently play a critical function in the diagnosis and management of these patients. Gout is a major concern globally and is the most common kind of inflammatory arthritis. The threat of both an initial gout attack and reoccurring gouty arthritis increases with uric acid levels. Hyperuricaemia, a prerequisite for gout, is related to the metabolic syndrome and is an independent danger factor for chronic kidney illness, stroke and perhaps ischaemic heart disease .It is connected with multiple damaging health results, including hypertension, heart disease and is involved as 
an important facet of the metabolic syndrome ${ }^{[6]}$. Furthermore, hyperuricaemia is currently being considered as a modifiable danger element for deterioration of renal function ${ }^{[7]}$. The management of gout is complexed by the episodic nature of the disease. In a populace research study on 2389 patients in South Australia, the general frequency of (self-reported) gout was $5.2 \%$ (males 8.5\%; women $2.1 \%$ ) ${ }^{[8]}$. Hyperuricaemia (uric acid $>0.42 \mathrm{mmol} / \mathrm{L}$ for men and $>0.34 \mathrm{mmol} / \mathrm{L}$ for women) existed in $17.8 \%$ of males and $15.4 \%$ of females. In the Framingham Heart Study, there was a degree reliant increase in both occurrence and related risk of creating gout pain with serum urate (see Figure 1) ${ }^{[9]}$. The possibility of persistent gouty arthritis also enhances with altitude of the serum uric acid level, with $\sim 35 \%$ of patients with an average uric acid level of $0.42 \mathrm{mmol} / \mathrm{L}$ having an attack 12 months or even more after an initial attack ${ }^{[10]}$. Decreasing the uric acid level below $0.36 \mathrm{mmol} / \mathrm{L}$ will result in the mobilisation of urate crystals out of joints and soft tissue. The reduced the serum urate level, the faster the resolution of the crystal deposition.

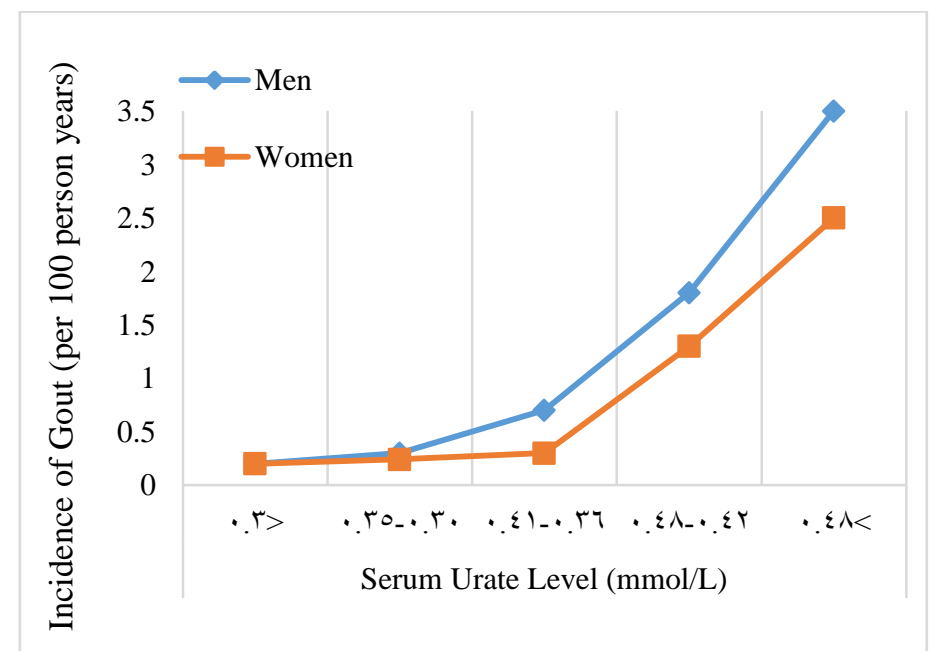

Figure 1. Incidence of initial gout attack based on uric acid level ${ }^{[9]}$.

\section{- Allopurinol in Treatment of Gout}

Allopurinol is a substrate for xanthine oxidase, which converts it to oxypurinol, which subsequently also inhibits xanthine oxidase. Allopurinol is metabolized in the liver and has a 1-3 hr half-life, while oxypurinol is eliminated in the pee and has a 12-17 hr half-life. As a result, allopurinol can be dosed daily, with lower dose demands in patients with kidney insufficiency, as the half-life of oxypurinol raises as the creatinine clearance lowers. The least expensive dosage that decreases the patient's serum urate to $<6 \mathrm{mg} / \mathrm{dl}$ ought to be used. The usual proposed dose is 300 $\mathrm{mg} /$ day, yet this cannot achieve a serum urate of $<6$ $\mathrm{mg} / \mathrm{dl}$ in $21-55 \%$ of individuals ${ }^{[11]}$. Up to $800 \mathrm{mg} /$ day could be called for. Lower doses might be efficient in patients with renal insufficiency, and slowly enhancing the dose of allopurinol to accomplish a serum urate $<6.0 \mathrm{mg} / \mathrm{dl}$ is not connected with an enhanced threat of negative effects or poisoning ${ }^{[12]}$. The abrupt reducing of serum urate after starting allopurinol or various other ULT typically causes acute gout. Preventative colchicine or NSAID is suggested, starting 2 weeks before allopurinol whenever feasible, and continuing for 3-6 months to protect against such attacks. An option is to start allopurinol at $50-100 \mathrm{mg} /$ day and boost by similar increments weekly up until the target serum urate is reached ${ }^{[12]}$.

Clinical teaching recommends that allopurinol needs not to be launched in the setting of an acute gout attack, since rapid lowering of serum urate may aggravate the assault. Delayed initiation might come at a price, as several patients never ever begin definitive urate-lowering treatment and are skeptical of chronic therapy after acute signs and symptoms settle. Because the introduction of allopurinol in 1964, reviews have attributed acute attacks of gout or worsening of continuous attacks to the beginning of allopurinol ${ }^{[13]}$. Inference could be indicated, because gout assaults continuously happen during the first couple of months after allopurinol is started and are symmetrical to the rate of uric acid reducing ${ }^{[14]}$. Concepts to clarify allopurinol-induced exacerbation of gout are controversial and do not have proof, yet they generally implicate urate concentration flux and conversion of tiny tophi ${ }^{[12]}$.

Recommendations have come to include complex guidelines for delayed and incremental initiation of definitive treatment, commencing after the acute attack has subsided, for both allopurinol and uricosurics ${ }^{[15]}$. Despite these well-intentioned guidelines, recent studies have implicated poor compliance, deficits in patient and physician knowledge, and complexity of present regimens requiring patients to return for graded increases of allopurinol as factors impeding better outcomes $^{[16]}$.If initiation of allopurinol could be simplified and administered in an adequate dose of 300 $\mathrm{mg}$ at the first medical encounter during an acute attack, then opportunity for education, improved outcomes, and cost containment might be realized. Evidence in support of delayed and stepped increase of allopurinol treatment is poor and supported by 2 studies describing case series ${ }^{[17]}$.No study has evaluated the initiation of allopurinol during attacks in patients with primary gout as they present in primary care settings, concomitantly treated with both indomethacin and colchicine. 
Prophylactic colchicine has been shown to reduce the frequency of gout flares in patients with interval gout and in patients beginning treatment with uricosuricsand allopurinol $^{[18]}$. Should allopurinol predispose to exacerbation, it is equally conceivable that the best time to initiate allopurinol would be during the acute attack, when patients also receive treatment doses of indomethacin and prophylactic doses of colchicine.

Urate lowering therapy is indicated in patients who have had an acute gout attack and whose uric acid level remains high, increasing the risk of a subsequent attack. The effect of allopurinol on uric acid level is dose dependent and variable. Graham et al examined blood levels of uric acid in 112 samples from patients taking various doses of allopurinol for at least one month $^{[19]}$. Doses of $300 \mathrm{mg}$ or more are required to achieve a $50 \%$ or more reduction in uric acid level. It is recommended that the dose of allopurinol be reduced in the presence of renal dysfunction in order to reduce the risk of serious adverse effects from accumulation of oxipurinol, the suspected toxic metabolite. There is little information available about the potential for reduced efficacy in this setting, but it would be expected that a lower dose would have lower efficacy. In addition, patients with a degree of renal dysfunction have reduced elimination of uric acid, further limiting the efficacy of xanthine oxidase inhibitors, which reduce production of uric acid. Studies of allopurinol used at an appropriately adjusted dose (based on creatinine clearance) have shown reduction of uric acid to target post-gout range $(<0.36 \mathrm{mmol} / \mathrm{L})$ in $\sim 20 \%$ of patients at three months and $\sim 40 \%$ of patients at $6-12$ months ${ }^{[19]}$.

Referrals have come to consist of complicated guidelines for postponed and step-by-step initiation of definitive treatment, starting after the acute attack has gone away, for both allopurinol and uricosurics ${ }^{[15]}$. Despite these well-intentioned guides, recent studies have implicated bad compliance, shortages in patient and doctor knowledge, and intricacy of present programs needing patients to return for graded boosts of allopurinol as factors hindering greater outcomes ${ }^{[16]}$.If initiation of allopurinol could be streamlined and administered in an adequate dosage of $300 \mathrm{mg}$ at the initial clinical encounter during an acute attack, after that chance for education, improved outcomes, and cost containment may be recognized. Evidence on behalf of delayed and pointered increase of allopurinol therapy is inadequate and sustained by 2 researches explaining case series ${ }^{[17]}$.

No research study has evaluated the initiation of allopurinol throughout attacks in patients with primary gout as they offer in primary care settings, concomitantly treated with both indomethacin and colchicine. Preventative colchicine has been shown to reduce the regularity of gout flares in patients with interval gout and in patients starting therapy with uricosurics and allopurinol ${ }^{[18]}$. Need to allopurinol incline to exacerbation, it is just as conceivable that the most effective time to start allopurinol would certainly be throughout the acute attack, when patients likewise receive treatment dosages of indomethacin and prophylactic dosages of colchicine.

Urate reducing treatment is indicated in patients who have had an acute gout attack and whose uric acid degree continues to be high, boosting the danger of a succeeding strike. The impact of allopurinol on uric acid level is dose reliant and changeable. Graham et al taken a look at blood degrees of uric acid in 112 samples from patients taking various dosages of allopurinol for at the very least one month ${ }^{[19]}$. Dosages of $300 \mathrm{mg}$ or more are required to accomplish a $50 \%$ or more decrease in uric acid level. It is advised that the dose of allopurinol be decreased in the presence of renal dysfunction in order to lower the danger of serious negative results from accumulation of oxipurinol, the believed hazardous metabolite. There is little information available about the potential for reduced effectiveness in this setting, but it would certainly be anticipated that a reduced dosage would certainly have lower efficiency. Additionally, patients with a degree of kidney disorder have lowered removal of uric acid, more limiting the efficiency of xanthine oxidase inhibitors, which reduce production of uric acid. Studies of allopurinol used at a properly readjusted dose (based on creatinine clearance) have shown reduction of uric acid to target post-gout variety $(<0.36 \mathrm{mmol} / \mathrm{L})$ in $\sim 20 \%$ of patients at 3 months and $40 \%$ of patients at $6-12$ months.

In the very first randomized, double-blind, placebo-controlled research ${ }^{[20]}$ of patients started on an adequate dose $(300 \mathrm{mg}$ ) of allopurinol throughout the acute gout attack, while all at once treated with indomethacin and colchicine, created remarkably similar declines in VAS and self identified gout flares, with narrow CIs. Although an ample dose of allopurinol is whatever dose is needed to achieve a serum urate listed below the objective of $6.0 \mathrm{mg} / \mathrm{dL}$, a starting dose of $300 \mathrm{mg}$ of allopurinol stays clear of the complicated tipped increments advised by guidelines ${ }^{[15]}$. Secondary end results, rate of erythrocyte sedimentation rate and C-reactive protein decrease, did not considerably vary between instant versus postponed teams.

All 26 patients in the immediate allopurinol therapy group showed a quick decrease in serum urate level, testimony to a high degree of compliance and 
ample urate change to evaluate the theory. Due to the fact that a quick reduction in serum urate is thought about to be an explanation for allopurinol-induced precipitation of gout attacks, we examined patients who in fact took allopurinol and returned for 10- and 30-day visits.

The number of self-reported acute attacks was low and comparable in both groups. All strikes remained in joints besides the primary joint, and only 1 was extreme enough to look for treatment. The rate of frequent gout strike was $10 \%$ over 1 month for the entire team, $8 \%$ in the allopurinol group, and $12 \%$ in the placebo team. All patients in these comparison groups likewise were taking preventative colchicine or nonsteroidal antiinflammatory drugs. The initial month $8 \%$ recurrent attack rate after allopurinol management during the acute attack contrasted favorably to that in the allopurinol groups in previous research studies ${ }^{[14]}(11.5 \%-13 \%)$ that did (surgery, diuretic initiation, injury), is cause for conclusive urate-lowering treatment. Therapy of the initial spontaneous strike is based upon the fact that $60 \%$ of people with a first attack experience a second flare within 1 year ${ }^{[21]}$.The price linked with gout flares and uncontrolled hyperuricemia is double the expense of gout flares with treated hyperuricemia $6.0 \mathrm{mg} / \mathrm{dL}$.Early streamlined treatment with allopurinol might mollify adverse effects from more comprehensive usage of nonsteroidal antiinflammatory drugs, restorative doses of colchicine, and new expensive treatments with febuxostat, pegloticase, anakinra, rilonacept, and canakinumab, implied for innovative cases of gout. Streamlined allopurinol administration in an extra complicated population with gout could better address issues of compliance and costeffectiveness.

There are epidemiologic suggestions that hyperuricemia is a mediator of a range of cardiovascular conditions ${ }^{[22]}$. Early initiation of allopurinol, for the initial gout assault, might enhance cardiovascular outcomes. In straightforward gout, all 3 medications, nonsteroidal anti-inflammatory medicine of selection, a prophylactic dosage of colchicine $0.6 \mathrm{mg}$ when daily, and appropriately dosed allopurinol $300 \mathrm{mg}$ daily, could be begun during the acute attack. Rated incremental dosing of allopurinol was not utilized since this study wished to reveal that the initiation of therapy can be done to advantage with an adequate $300 \mathrm{mg}$ dose, saving unneeded visits, cost, chronic under dosing, and complexity of therapy ${ }^{[22]}$.

\section{- Adverse Effects of Allopurinol}

Allopurinol could lower the number of blood cells in your body that generate blood clot and that assist you deal with infections, which can make it simpler for you to hemorrhage from an injury or get ill when revealed to others that are ill. You need to prevent being around individuals who are sick or have infections while taking this medicine.

About 20\% of patients on allopurinol report negative effects and 5\% discontinue, the commonest being gastrointestinal intolerance and body skin rash. If the rash is light, allopurinol can be kept and the patient rechallenged after the rash has cleared. Desensitization methods for allopurinol are efficient in some patients, yet troublesome ${ }^{[23]}$. Various other negative reactions include fever, poisonous skin necrolysis, alopecia, bone marrow suppression, granulomatous liver disease, jaundice, sarcoid-like response, and vasculitis. Allopurinol hypersensitivity disorder is a dreaded serious reaction that consists of fever, skin rash, eosinophilia, hepatitis, progressive renal failing, and death as a result of multi-organ vasculitis.Individuals with pre-existing renal insufficiency or on diuretics go to best danger. The threat of allopurinol hypersensitivity might associate finest with the starting dose, which dosage needs to be decreased based upon creatinine clearance ${ }^{[23]}$.

\section{- Drug Interaction with Allopurinol}

There are fairly a couple of medication- drug communications with allopurinol, however a number of medications are suspended by xanthine oxidase, consisting of azathioprine and 6-mercaptopurine, and their degrees may come to be poisonous in the visibility of allopurinol. Allopurinol may diminish hepatic microsomal drug-metabolizing enzyme task causing longer half-lives of warfarin and theophylline. Concomitant ampicillin might boost the danger of rash and cyclophosphamide the danger of bone marrow suppression with allopurinol ${ }^{[24]}$.

It is the raised possibility of this potentially dangerous unfavorable result that has caused the renal function-based dosage recommendations for allopurinol. Commencement of allopurinol ought to as a result be with a reduced dose, typically no more than $100 \mathrm{mg}$ daily. Slow increases may be made at regular monthly periods according to feedback, going for a plasma urate concentration. Two crucial aspects which harm the efficiency of allopurinol are inadequate adherence and sub-optimal dosing to adequately lower urate levels ${ }^{[25]}$. Educating and empowering patients in the control of their own condition might boost subsequent administrations, such as use allopurinol .It is clear that patients have to be better notified of the long-lasting advantages of allopurinol, such as avoiding 
irreparable joint damage and difficulties related to comorbidities $^{[25]}$.

\section{CONCLUSION}

Allopurinol is a type of drug called a xanthine oxidase inhibitor. It functions by lowering the production of uric acid in the body. It can be found in the kind of an oral tablet. Generalist physicians, specifically general internists and primary care physicians, are usually the first to see individuals with gout and for that reason play an essential role in the diagnosis and management of these patients. Gout is mostly managed in primary care and lasting treatment aims to reduce serum uric acid degrees with using allopurinol. Urate lowering therapy is indicated in patients who have had an acute gout attack and whose uric acid level remains high, increasing the risk of a subsequent attack. The effect of allopurinol on uric acid level is dose dependent and variable.

To conclude, allopurinol-induced adverse events create more frequently in patients who take colchicines or statins. A mindful method needs to be taken when prescribing allopurinol to these risky teams. Two essential factors which hinder the performance of allopurinol are poor adherence and sub-optimal application to adequately lower urate levels.Informing and equipping patients in the control of their very own disease might enhance succeeding monitorings, such as use of allopurinol. It is certain that patients have to be much better educated of the long-lasting advantages of allopurinol, such as preventing irreversible joint damage and complications related to comorbidities.

\section{REFERENCES}

1. Neogi T(2011): Clinical practice: gout. N Engl J Med., 364:443452.

2. Tausche AK, Aringer M, Schroeder HE et al. (2008): The Janus faces of allopurinol-allopurinol hypersensitivity syndrome. Am J Med ., 121:e3-e4.

3. Hung CC, Liu WC, Kuo MC et al.(2009): Acute renal failure and its risk factors in Stevens-Johnson syndrome and toxic epidermal necrolysis. Am J Nephrol . ,29:633-638.

4. Arellano F, Sacristan JA(1993): Allopurinol hypersensitivity syndrome: a review. Ann Pharmacother., 27:337-343

5. Hande KR, Noone RM, Stone WJ (1984): Severe allopurinol toxicity: description and guidelines for prevention in patients with renal insufficiency. Am J Med., 76:47-56.

6. Abeles $\mathbf{A M ( 2 0 1 5 ) : H y p e r u r i c a e m i a , ~ g o u t ~ a n d ~ c a r d i o v a s c u l a r ~}$ disease: an update. CurrRheumatol Rep., 17(13).

7. Kanji T, Gandhi M, Clase CM, Yang R (2015): Urate lowering therapy to improve renal outcomes in patients with chronic kidney disease: systematic review and meta-analysis. BMC Nephrology, 16(1): 58 .

8. Ting K, Gill TK, Keen H, Tucker GR, Hill CL(2016): Prevalence and associations of gout and hyperuricaemia: results from an Australian population-based study. Internal medicine journal, 46(5):566-73.
9. Roddy E, Choi H (2014):Epidemiology of gout. Rheum Dis Clin North Am., 40(2): 155-175

10. Shoji A, Yamanaka H, Kamatani N(2004): A retrospective study of the relationship between serum urate level and recurrent attacks of gouty arthritis: evidence for reduction of recurrent gouty arthritis with antihyperuricemic therapy. Arth Care and Research, 51(3):321-5.

11. Li-Yu J, Clayburne G, Sieck M,Beutler A, Rull M, Eisner E et al. (2001): Treatment of chronic gout. Can we determine when urate stores are depleted enough to prevent attacks of gout? J Rheumatol., 28: 577-580.

12. Dalbeth N, Stamp $\mathbf{L}$ (2007): Allopurinol dosing in renal impairment: walking the tightrope between adequate urate lowering and adverse events. Semin Dial., 20: 391-395.

13. Neogi T(2011): Clinical practice. Gout. N Engl J Med., 364:443452.

14. Sundy JS, Becker MA, Baraf HS et al. (2008): Reduction of plasma urate levels following treatment with multiple doses of pegloticase (polyethylene glycol-conjugated uricase) in patients with treatment-failure gout: results of a phase II randomized study. Arthritis Rheum., 58:2882-2891.

15. Jordan KM, Cameron JS, Snaith M et al. (2007): British society for rheumatology and British health professionals in rheumatology guideline for the management of gout. Rheumatology (Oxford). , 46:1372-1374.

16. Mikuls TR, Farrar JT, Bilker WB, Fernandes S, Saag KG (2005): Suboptimal physician adherence to quality indicators for the management of gout and asymptomatic hyperuricaemia: results from the UK General Practice Research Database (GPRD). Rheumatology (Oxford), 44: 1038-1042.

17. Yue TF, Gutman AB(1964): Effect of allopurinol (4hydroxypyrazolo-(3,4- D)pyrimidine) on serum and urinary uric acid in primary and secondary gout. Am J Med., 37:885-898.

18. Borstad GC, Bryant LR, Abel MP, Scroggie DA, Harris MD, Alloway JA(2004): Colchicine for prophylaxis of acute flares when initiating allopurinol for chronic gouty arthritis. J Rheumatol., 31:2429-2432.

19. Wright DF, Duffull SB, Merriman TR, Dalbeth N, Barclay ML, Stamp LK(2016): Predicting allopurinol response in patients with gout. British journal of clinical pharmacology, 81(2):277-89.

20. Taylor TH, Mecchella JN, Larson RJ, Kerin KD, MacKenzie TA(2012): Initiation of allopurinol at first medical contact for acute attacks of gout: a randomized clinical trial. The American journal of medicine, 125(11):1126-34.

21. Neogi T, Hunter DJ, Chaisson CE, Allensworth-Davies D, Zhang Y(2006): Frequency and predictors of inappropriate management of recurrent gout attacks in a longitudinal study. $\mathrm{J}$ Rheumatol., 33:104-109.

22. Seth R, Kydd AS, Buchbinder R, Bombardier C, Edwards CJ(2013): Allopurinol for chronic gout. Cochrane Database Syst Rev., 4.

23. Burns CM, Wortmann RL (2012): Latest evidence on gout management: what the clinician needs to know. Therapeutic advances in chronic disease., 3(6):271-86.

24. Blaker PA(2017): The Mechanism and Drug InteractionAllopurinol and Azathioprine and Risk of Bone Marrow Suppression. Can be retrieved from https://www.ebmconsult.com/articles/allopurinol-azathioprineinteraction-mechanism-wbc

25. Kuo C, Grainge MJ, Mallen C, Zhang W, Doherty M (2015): Rising burden of gout in the UK but continuing suboptimal management: a nationwide population study. Ann Rheum Dis., 74:661-7. 